A R R T I I C U L L O I $N V E S T I$ G A C I Ó $N$ C I E E N

TEC C N O L Ó G I C C A

\title{
SOPORTE SOCIAL Y FAMILIAR: EXPERIENCIAS CON FAMILIAS DE PACIENTES ATENDIDOS EN UNA CLINICA UNIVERSITARIA
}

\author{
FAMILY AND SOCIAL SUPPORT: EXPERIENCIES WITH PATIENTS \\ FAMILY MEMBERS WHO WERE ASSISTED AT A UNIVERSITY CLINIC
}

* Trabajadora Social Magister en Terapia Familiar docente titular de la Escuela de Ciencias Sociales y coordinador académica de los Posgrados en Familia de la Universidad Pontificia Bolivariana. Miembro del grupo de Investigación en Familia de la Universidad Pontifici Bolivariana.

**Trabajadora Social Clínica Universitaria Bolivariana, Magister en Terapia de Familia. Líder Grupo de Dolor y Cuidados Paliativos de

laniversidad

Pontifi-

cia Bolivariana.
${ }^{1}$ Participaron como auxiliares de investigación, las estudiantes de Trabajo Social de la Universidad Pontificia Bolivariana, Adriana Maria Duque Palacio y Lina Marcela Rojas Monsalve.
Por: María Eugenia Agudelo Bedoya*

María Clara Vélez**

Recibido: 2 de diciembre de 2015 - Aprobado: 27 de julio de 2016

\section{RESUMEN}

Este artículo está basado en una investigación realizada sobre problemáticas familiares de 133 pacientes interconsultados y atendidos por Trabajo Social en los servicios de internación de la Clínica Universitaria Bolivariana (CUB) entre febrero-julio de 2013. ${ }^{1}$ Se utilizó un diseño mixto, integrando aspectos cuantitativos y cualitativos para describir las familias, variables de la atención ofrecida por trabajo social y la manera como se organizaban las familias para el cuidado del paciente en su hospitalización. Se encontraron varias configuraciones familiares, un porcentaje alto atravesando ciclo vital de adulto o anciano. El mayor número de remisiones corrieron por cuenta del médico internista y correspondieron a situaciones catalogadas por trabajo social como "problemas relacionados con otros hechos estresantes que afectan la familia". El tipo de intervención en el que fueron atendidas fue el acompañamiento. Algunas de las familias, encontraron soporte entre sus miembros, amigos, vecinos, cuidadores formales e informales y en instituciones que por su competencia deben brindarlo. La ausencia de estos apoyos se constituye el principal motivo de remisión a trabajo social.

Palabras clave: Trabajo Social, Familia, Enfermedad, Intervención, Soporte familiar y Soporte social.

\begin{abstract}
This article is based on a research conducted about family problematics of 133 interconsulted patients and seen by Social Work in the sections of inpatient services of the Clinica Universitaria Bolivarana, from February to July 2013. A mixed design was used, integrating quantitative and qualitative aspects to describe the families assisted, variables of the care serviced offered by Social Work section and the way families were organized to recieve the care service while in their hospitalization. Various family conformations were found with a high percentage of life cycle of adult or elderly people. The largest number of referrals was provided by the internist and corresponded to situations cataloged by Social Work as "problems related to other stressful events affecting the family." The type of intervention that was addressed was the companion program. Some families found support among their members of the family, friends, neighbors, formal and informal caregivers and institutions that by its jurisdiction must provide it. The absence of these supports becomes the main reason for referral to social work.
\end{abstract}


Keywords: Social Work, Family, Disease, Intervention, Family Support and Social Support

\section{Introducción}

a familia como sistema relacional, afronta diferentes experiencias
conectadas con su proceso evolutivo, su dinámica interna, sus
funciones sociales y con situaciones inesperadas, entre ellas, el proceso
de enfermedad que puede vivir alguno de sus miembros.

Existen algunas enfermedades o fases de la misma que requieren de un proceso de atención en un servicio de internación hospitalaria, es decir, atención en cuidados intensivos o en hospitalización, con el fin de buscar la estabilidad física y mejorar la calidad de vida del paciente. En este contexto cada vez se proclama más la importancia de la atención integral e interdisciplinaria, haciendo énfasis en que no solo se enferma el paciente, sino también la familia, pues participa de todos los aspectos y actividades que incluye dicha experiencia. Como sistema primordial de cuidado para el enfermo, la cotidianidad del cuidado en la institución de salud le exige cambios a diferentes niveles, que son vividos en ocasiones con flexibilidad y fluidez, en otras en cambio, con conflictos y sufrimiento.

En la Clínica Universitaria Bolivariana el equipo de salud está integrado por médicos especialistas, enfermeras, auxiliares de enfermería, fisioterapeutas, terapeutas respiratorios, psicóloga y trabajadoras sociales. Los pacientes y las familias que son derivados para atención de trabajo social, llegan a este servicio remitidas por un médico especialista u otro profesional del área de la salud que identifica situaciones familiares que considera pueden ser abordadas por trabajo social para ayudarlas a ser un soporte social efectivo que favorezca la adaptación a la situación de enfermedad o la superación de una crisis familiar.

Ligado a lo anterior, surge el interés desde Trabajo Social por describir las problemáticas familiares de pacientes interconsultados y atendidos por dicha área en los servicios de internación de la Clínica Universitaria Bolivariana entre febrero y julio de 2013, identificando: la dificultad más común, algunas características sociofamiliares, la intervención de trabajo social y analizando las narraciones consignadas por las trabajadoras sociales respecto a lo que dicen las familias sobre cómo se organizan, para responder a las necesidades de cuidado del paciente. 


\section{Metodología}

T a investigación se desarrolló combinando los enfoques cualitativo y cuantitativo, lo que permitió presentar tanto datos numéricos como contextuales de las problemáticas familiares en el ámbito hospitalario, de tal manera que se pudo evidenciar en los pacientes remitidos a Trabajo Social, las problemáticas familiares que presentaron, la descripción de como las familias se organizan para resolver dichas problemáticas y el nivel de intervención de trabajo social en el que fueron abordadas.

Siendo las experiencias de las familias fenómenos sociales, que desde el punto de vista sistémico suelen ser impredecibles, dinámicas, autorreferenciales y contextuales, esta investigación se apoyó en una relación de complementariedad entre los enfoques. Refiere al respecto Galeano (2004) que:

"La realidad social comporta dimensiones posibles y necesarias de cuantificar y dimensiones que es significativo cualificar, unas y otras hacen parte de un mismo proceso y su diferenciación solo obedece a criterios de carácter metodológico. Las dimensiones cuantitativas permiten establecer indicadores, índices, proyecciones, caracterizar variables que son básicas para el diagnóstico y la sustentación de políticas sociales. Las dimensiones cualitativas aportan a la comprensión de razones, lógicas, racionalidades, visiones, modos de ser y de comportarse que llenan el dato de contenido y permiten, desde los múltiples actores sociales, conocer la diversidad y heterogeneidad social". Pag 25

El tema objeto de esta investigación se enmarcó en la visión holística-sistémica de la enfermedad como una experiencia humana, en donde se conectan diferentes dimensiones del ser, tales como: la física, psicológica, espiritual y relacional. El alcance fue descriptivo y las unidades de análisis fueron 133 pacientes y sus familias.

Desde el punto de vista cuantitativo el estudio fue transeccional y las variables que se tomaron en el reporte estadístico fueron: características sociodemográficas de los pacientes (procedencia, edad, sexo, nivel educativo, ocupación, estrato, rol en la familia), características de las familias (tamaño, tipo y etapa del ciclo vital familiar), problemáticas familiares más frecuentes y porcentaje de especialidades médicas que más interconsultaron al área de Trabajo Social por situaciones relacionadas con dificultades familiares y nivel de intervención realizado por dicha área. Con respecto a lo cualitativo 
se realizó un ejercicio hermenéutico de interpretación, sobre las narraciones consignadas por Trabajo Social en la historia clínica acerca de las relaciones familiares en torno al diagnóstico médico.

Para recolectar la información se elaboró una guía de entrevista para interrogar la historia clínica de cada paciente, en donde se recogieron los datos cuantitativos y se incluyeron preguntas abiertas sobre las relaciones familiares. Los datos recogidos en la guía se depositaron en un cuadro y se usó Excel para el análisis estadístico. La información cualitativa, se recopiló mediante una matriz y se utilizó Atlas Ti.

\section{Hallazgos}

A continuación se presentan los hallazgos, abordando en primer lugar los aspectos cuantitativos $y$, en segundo lugar, se expone la información de índole cualitativa.

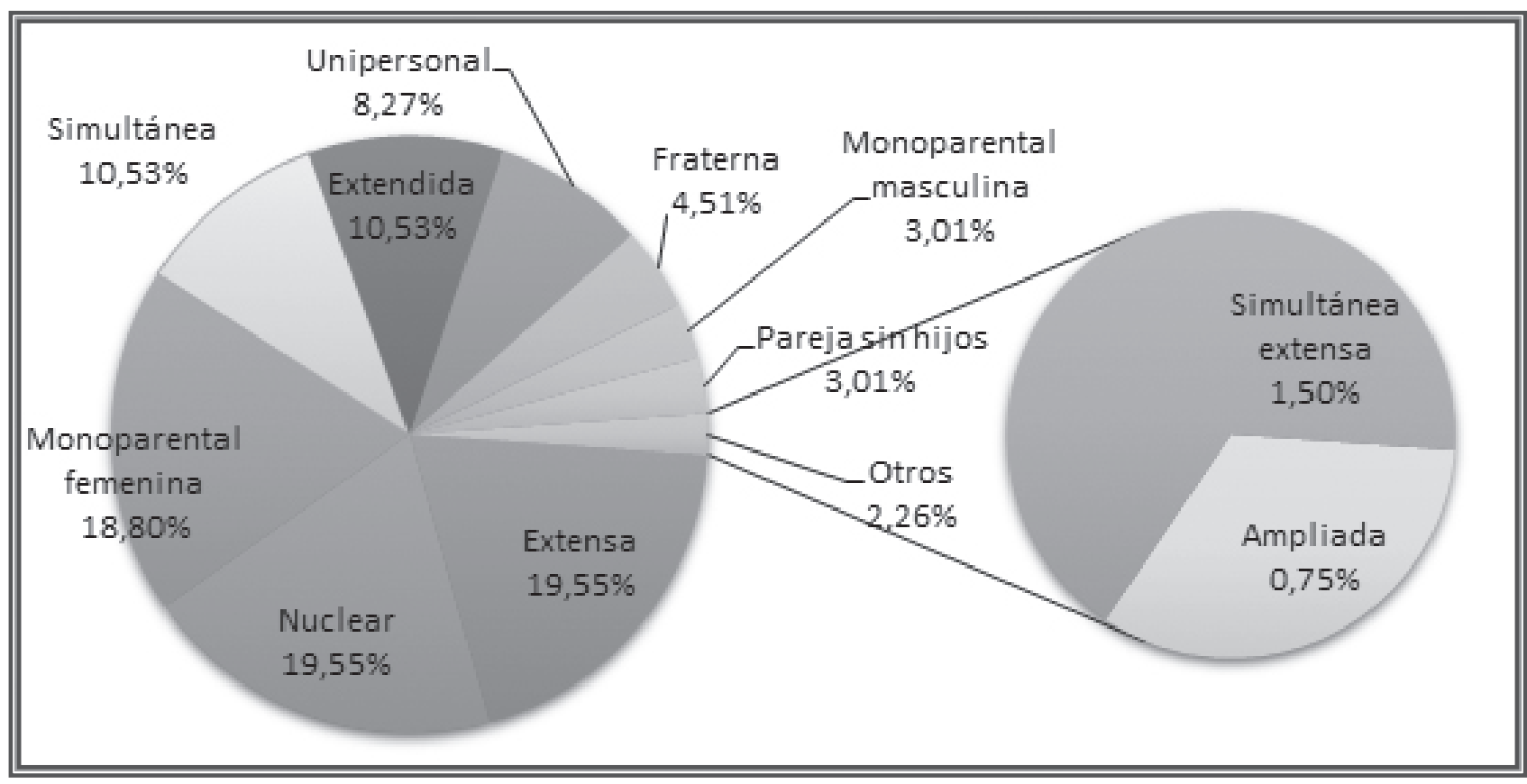

Figura 1. Descripción de las familias. Distribución según la tipología familiar Fuente: Elaboración de las investigadoras

Acogiendo la clasificación de Estrada (2006 ), en la gráfica se observa que hay presencia de una variedad de tipologías familiares muy amplia, resaltando que la familia nuclear aparece con un porcentaje igual a la extensa y muy similar a la monoparental femenina. Tanto la simultánea como la extendida obtienen también porcentajes iguales e importantes. Este hallazgo coincide con la evidencia de que la familia en nuestro medio sigue su constante dinamismo 


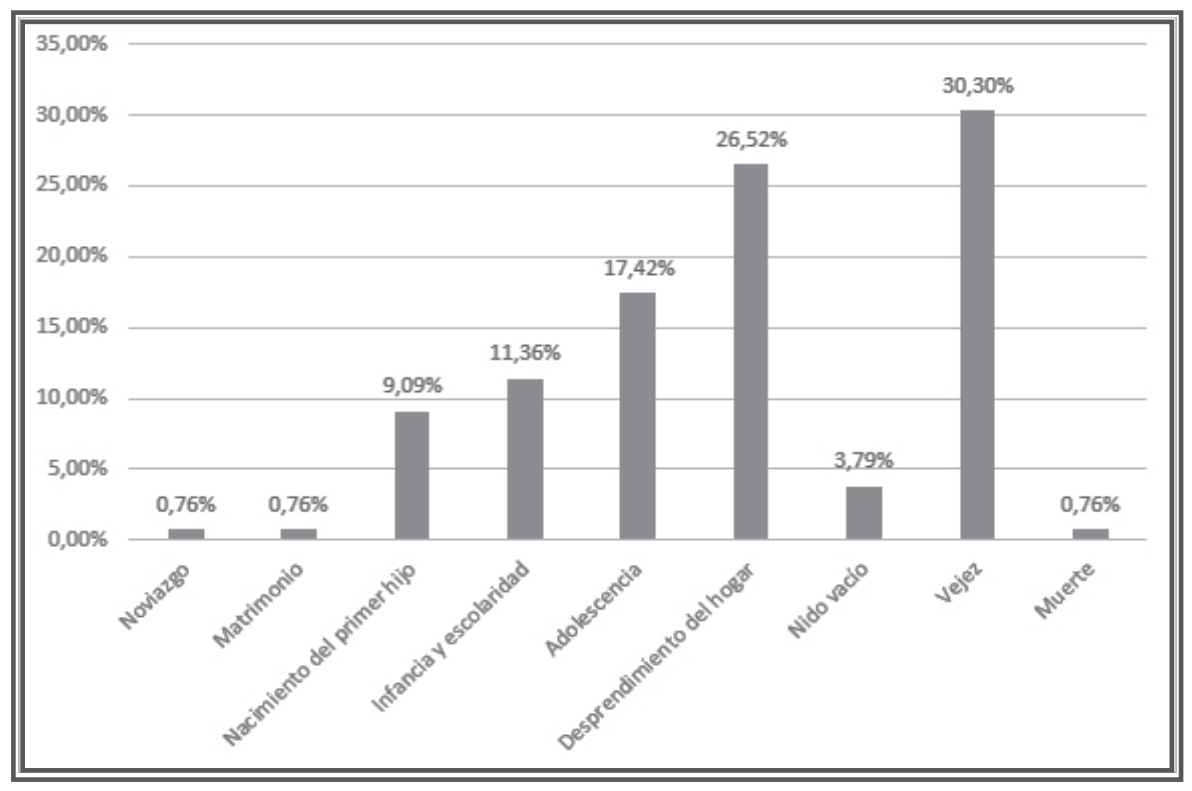

Figura 2. Distribución según el ciclo vital familiar Fuente: Elaboración de las investigadoras

y transformación y concuerda con lo hallado en la Encuesta Nacional de Salud (Flórez, 2010) según la cual 35\% son hogares nucleares completos, $17 \%$ extensas, y $12 \%$ nucleares incompletos (monoparentales).

La distribución en las diferentes etapas del ciclo vital familiar permite establecer que es mayor el número de las familias que se encuentran en la etapa de la vejez, donde se espera una organización que permita apoyar a los adultos mayores en el proceso que viven, con requerimientos de acompañamiento y cuidado especiales, ligados a condiciones normales de deterioro o afecciones de salud. Le siguen las familias en etapas iniciales o intermedias del ciclo vital, en las cuales, la familia debe disponer de sus recursos y mecanismos de organización para enfrentar condiciones que suponen los diferentes cambios que atraviesan en todo el curso de su desarrollo, los cuales, pueden tornarse más estresantes cuando en las familias hay alguien con

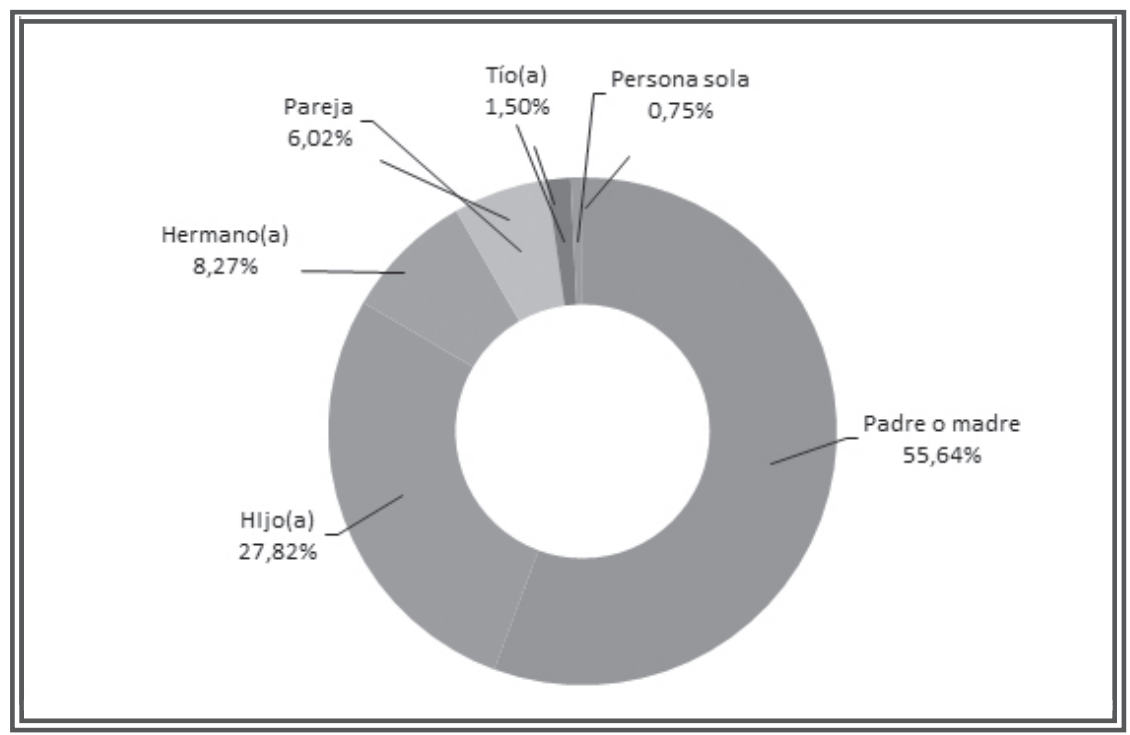

Figura 3. Distribución según el rol que ocupa el paciente dentro de la familia Fuente: Elaboración de las investigadoras problemas de salud.

Según el rol en la familia, son el padre o la madre quienes obtienen el mayor porcentaje, correspondiendo a más de la mitad de la población abordada. Esto es coincidente tanto con la edad de los pacientes como con el ciclo vital familiar, ya que se trata de una porción significativa de adultos y adultos mayores. En nuestra cultura, padre $\mathrm{y}$ madre $\mathrm{y}$, principalmente 
esta última, suele congregar la familia en torno a sí, por lo cual, sería de esperar que ante los requerimientos de cuidados en salud, las familias se dispusieran a ofrecerles apoyo y atención, cuando no lo hacen, se configura una crisis frente al cuidado del paciente, que se convierte en motivo de consulta a profesionales, como en este caso. De igual forma, los hijos generalmente cuentan en primera instancia con sus padres para ayudarlos en las necesidades y tramites que implica un tratamiento médico.

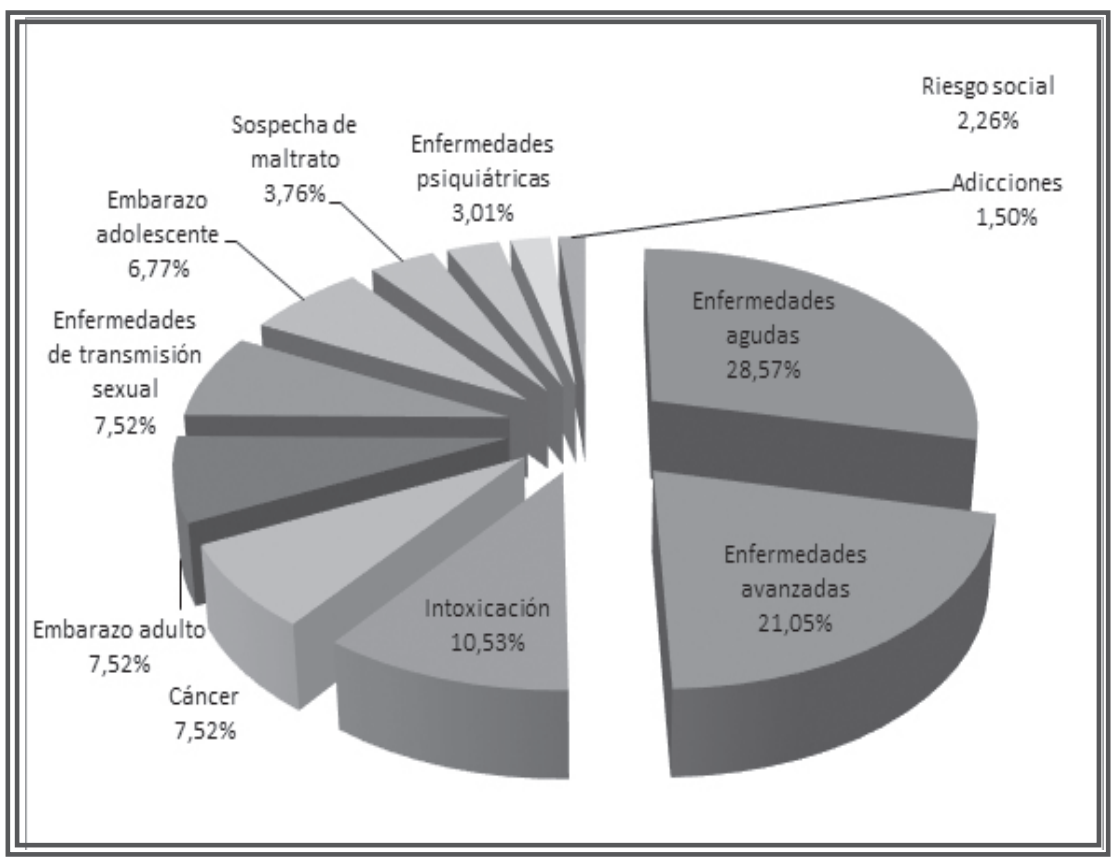

Figura 4. Distribución según el diagnóstico médico Fuente: Elaboración de las investigadoras

En la gráfica, se evidencia que la mayoría de los pacientes sufrían enfermedades agudas, es decir, aquellas que son tratables, como neumonía, infección del tracto urinario, entre otras. Con un porcentaje del 21,05\%, se resaltan las enfermedades avanzadas, que hacen referencia a fases terminales o fallas multiorgánicas. Le sigue, con un 10,53\%, la intoxicación, la cual en estos casos está asociada a intentos de suicidio por parte de los pacientes identificados. Con igual porcentaje (7.52\%), aparecen el cáncer, el embarazo adulto y las enfermedades de trasmisión sexual. En menores porcentajes se evidencia el embarazo adolescente, la sospecha de maltrato (que reúne todos aquellos abusos sexuales, psicológicos o físicos y también los ocurridos por negligencia), las enfermedades psiquiátricas, el riesgo social y las adicciones.

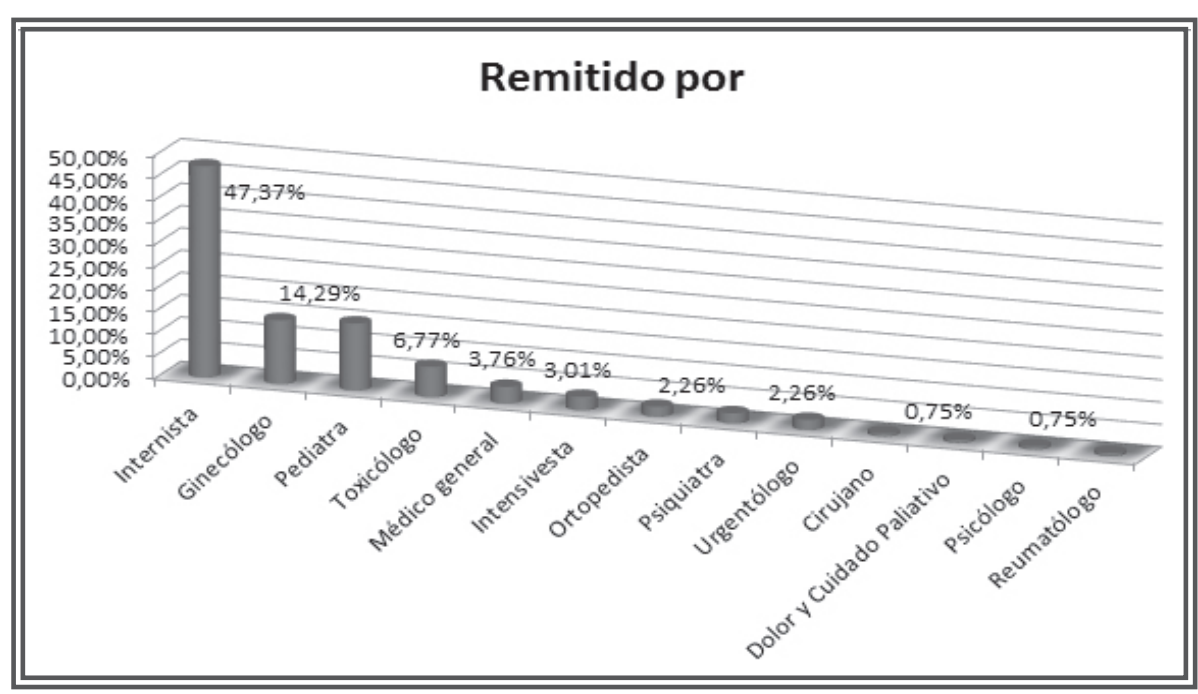

Figura 5. Distribución según la especialidad médica que remite a trabajo social Fuente: Elaboración de las investigadoras 
Según el registro en las historias clínicas revisadas, es el médico internista quien mayor número de remisiones efectúa para interconsulta con trabajo social lo que puede explicarse teniendo en cuenta que es la especialidad que permanece tiempo completo en la clínica. Como se aprecia en la gráfica hay otros especialistas que interconsultan lo que da cuenta del impacto que le atribuyen a los factores sociofamiliares de la enfermedad en el paciente y su familia.

\section{Distribución según el diagnóstico de Trabajo Social}

1 CIE 10 (Clasificación Internacional de Enfermedades), publicado por la Organización Mundial de la Salud, es un referente estadístico internacional que permite clasificar y codificar, entre otras variables, las enfermedades, las causas externas de daño y las circunstancias sociales, que pueden favorecer la perturbación de la enfermedad, o en el caso de la valoración

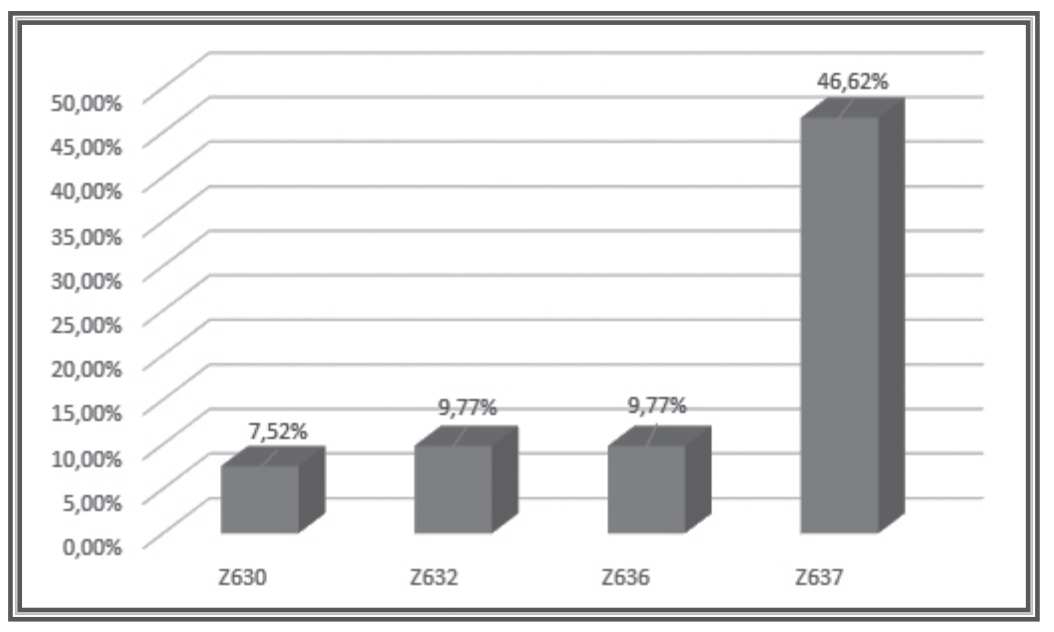

por Trabajo Social, la alteración en las relaciones o dinámicas familiares. En el pie de página² se muestran los diagnósticos utilizados por Trabajo Social en la Clínica Universitaria Bolivariana dentro del periodo analizado.

Las siguientes gráficas permiten identificar la distribución de los diagnósticos según los datos recogidos en las historias clínicas.

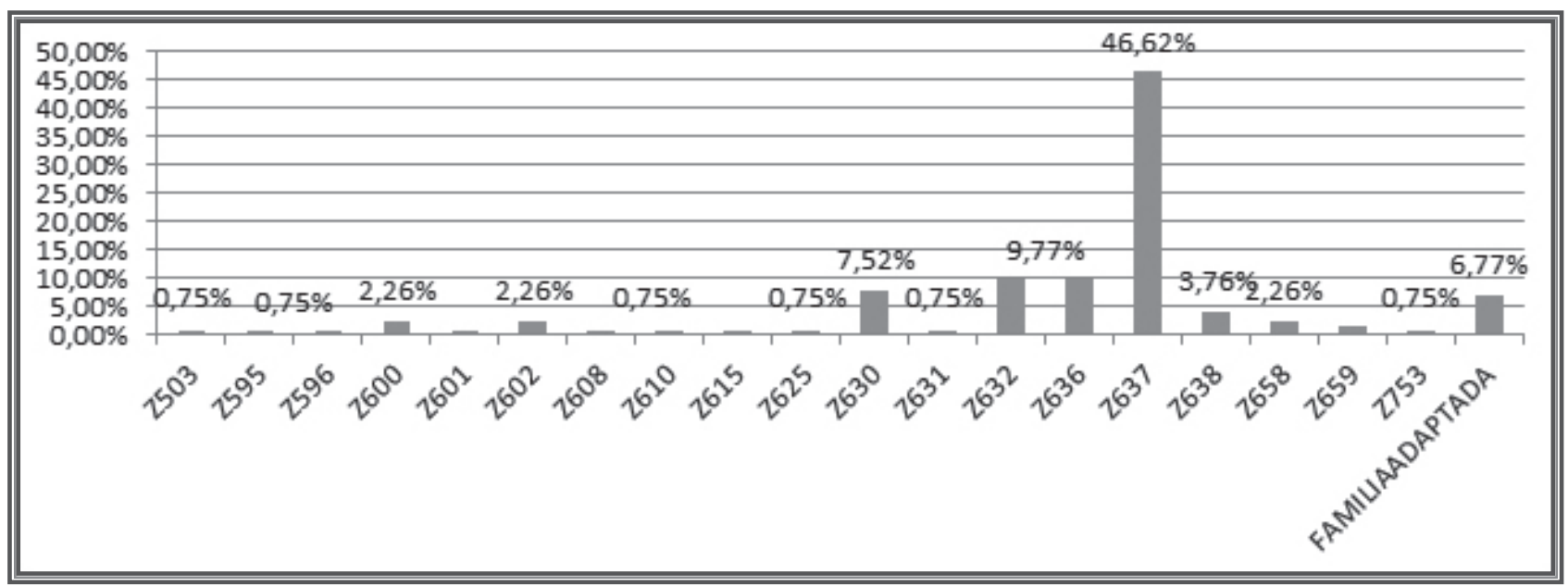

Figura 6. Distribución según el diagnóstico de trabajo social Fuente: Elaboración de las investigadoras 
Al visualizar la distribución del diagnóstico de trabajo social, se puede decir que el $\mathbf{7 3 . 6 \%}$ de las familias se ubican en cuatro categorías diagnósticas que se especifican en la siguiente gráfica. Cabe anotar que casi el 7\% se registran como familias sin problemáticas familiares relacionadas con la enfermedad y se denotan como adaptadas.

El mayor porcentaje corresponde a la categoría "problemas relacionados con otros hechos estresantes que afectan la familia" (Z637) en la cual se incluye una gama muy amplia de situaciones estructurales o críticas que alteran el ambiente y las interacciones de la familia, principalmente, el hecho de tener un ser querido enfermo, que puede generar cambios en la estructura relacional y comportamental. En segundo lugar aparecen, con igual peso, los diagnósticos "problemas relacionados con el apoyo familiar inadecuado" (Z632) y "problemas relacionados con familiar dependiente de cuidado" (Z636), es decir, que la enfermedad y el enfermo generan tensiones ligadas a demandas físicas, emocionales, afectivas y económicas, que pueden cargar de tensión la vida familiar y generar alteraciones en las relaciones de sus miembros, lo cual puede afectar a cada uno y a todos como totalidad. El tercer lugar, corresponde al diagnóstico "problemas en la relación entre esposos o pareja" (Z630), subsistema este bastante importante para sus miembros y muy susceptible ante las tensiones, las cuales pueden incluso, poner a prueba la estabilidad y perdurabilidad del vínculo.

\section{Tipo de intervención del Trabajador Social}

omo se observa en la siguiente gráfica, el mayor porcentaje corresponde a las familias que fueron atendidas por Trabajo Social en una sesión, le siguen las familias que estuvieron entre 2 y 4 sesiones y por último, las que tuvieron entre 5 y 7 citas, distribución que está ligada al tiempo de permanencia del paciente en la clínica, el cual es definido por el médico tratante. Una vez el paciente es dado de alta, son pocas las familias que continúan en proceso terapéutico con Trabajo Social. De ahí el interés de ofrecerles una atención integral durante el tiempo que dura la hospitalización del paciente.

Si bien se tiende a considerar que a más número de citas en las que participa una familia el beneficio es mayor, es importante aclarar que en el ámbito hospitalario, donde se abordan situaciones tan diversas, puede ser más importante y esclarecedor el contenido y la intensidad de la conversación que la familia sostiene con el profesional que el número de veces, generalmente los tiempos de interconsulta en hospitalización son reducidos debido a que el proceso de recuperación dentro de la clínica también lo es.
${ }^{2}$ Z502: Rehabilitación del alcohólico; Z503: Rehabilitación del drogadicto; Z595: Problemas relacionados con pobreza extrema; lacionados con bajos ingresos: 7600 : Proble ingresos, Z600: Problemas relacionados con e ajuste a las transiciones
del ciclo vital; Z601: Problemas relacionados con situación familiar atípica; Z602: Problemas relacionados con persona que vive sola; Z608: Otros problemas relacionados con el mas relacionados con el Problemas relacionados con la pérdida de la relación afectiva en la infancia; Z613: Problemas relacionados blemas relacionados con eventos que llevaron a la pérdida de la autoestima; Z615: Problemas relacionados con el abuso sexual del niño por persona ajena; Z625: Otros problemas relacionados con negligencia en la crianza del niño; Z630: Problemas en la relación entre esposo o pareja; Z632: Problemas relacionados con el apoyo familiar inadecuado; Z636: Problemas relacionados con familiar dependiente, necesitado de cuidate, necesitado de cuidaestresantes que afectan la familia; Z638: Otros problemas especificados relacionados con el grupo familiar priproblemas relacionados con circunstancias dos con circunstancias
psicosociales; Z659: Problemas relacionados con circunstancias psicosociales no específicas; Z753: Problemas relaciona mas relacionados con disponible o inaccesible; Familia adaptada: Este ítem no aparece en el CIE 10 , es un término que se ajustó en la institución para describir que no se percibe ninguna problemática familiar ni social, que afecte la recuperación adecuada del paciente. 


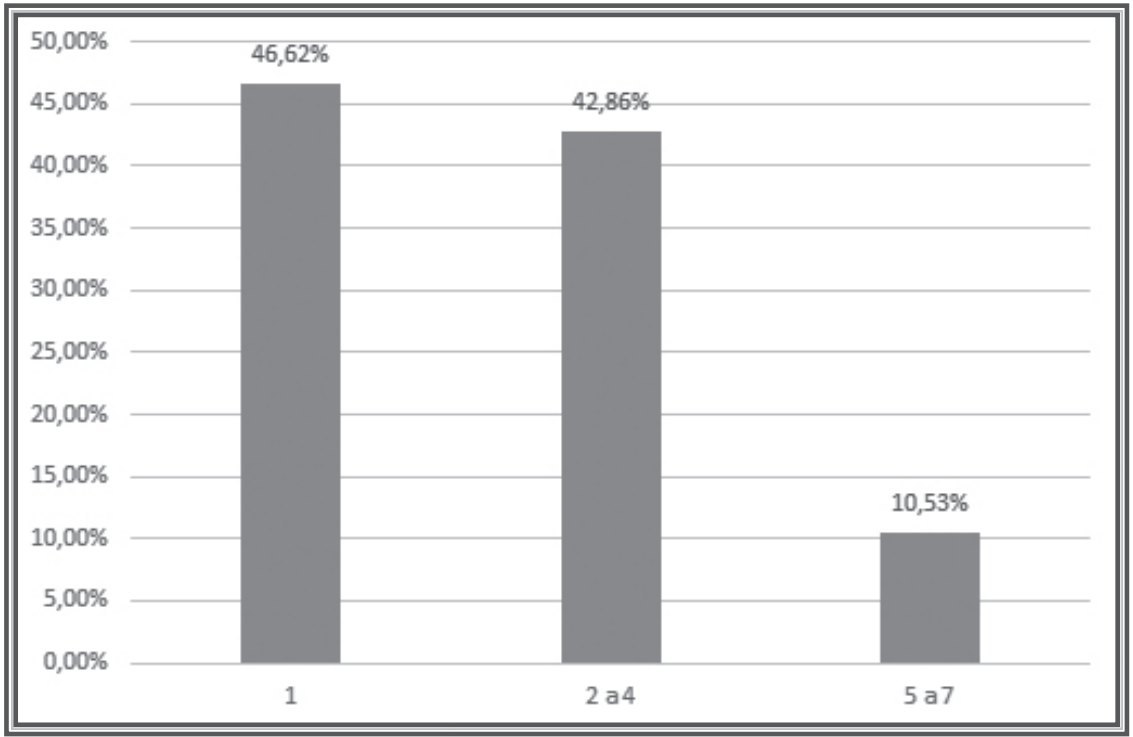

Figura 7. Distribución según el número de sesiones realizadas Fuente: Elaboración de las investigadoras
En todos los casos que llegan a Trabajo Social se realiza inicialmente una evaluación de la problemática familiar y a partir de esta, se define el nivel de intervención a efectuar. Cada uno de estos niveles corresponde a situaciones y objetivos diferentes, siendo todos ellos igualmente importantes, los cuales requieren una preparación sólida del profesional que los atiende. A continuación, se describen las particularidades de cada uno de ellos, acogiendo la clasificación que nos presenta Laura Fruggeri (2002, p.7):

- Acompañamiento o sostén: Se refiere a las necesidades que las familias experimentan en los eventos críticos inesperados. Se incluyen las intervenciones de los servicios sanitarios que ponen a disposición competencias terapéuticas para superar enfermedades y competencias de rehabilitación para reducir el daño producido por problemas de salud abarcando tanto al paciente como a su familia y algunas veces activando las redes de apoyo para estos.

- Facilitación: En esta categoría se incluyen todas las acciones relacionadas con las tareas evolutivas previstas en las diversas etapas del ciclo vital familiar. Esta intervención tiene como objetivo amplificar los recursos internos de las familias, en los niveles emocional, afectivo, espiritual, económico e interaccional que favorece el proceso de adaptación de la familia.

- Mediación: Son intervenciones utilizadas en fases específicas de transición, en particular en aquellas donde los implicados no logran encontrar una solución negociada en forma autónoma y acuden a un tercero, en este caso el trabajador social.

- Control: Son las intervenciones, solicitadas por la familia o determinadas por un agente externo, que se activan cuando esta presenta problemas de violencia, 
abuso o incapacidad grave para asumir las tareas de protección de sus miembros.

- Terapia: Este nivel de intervención tiene lugar en los casos en los que se manifiesta un malestar psicopatológico o problemas relacionales estructurales. Puede estar dirigida a una sola persona o a todo el grupo familiar $y$, en ambos casos, se

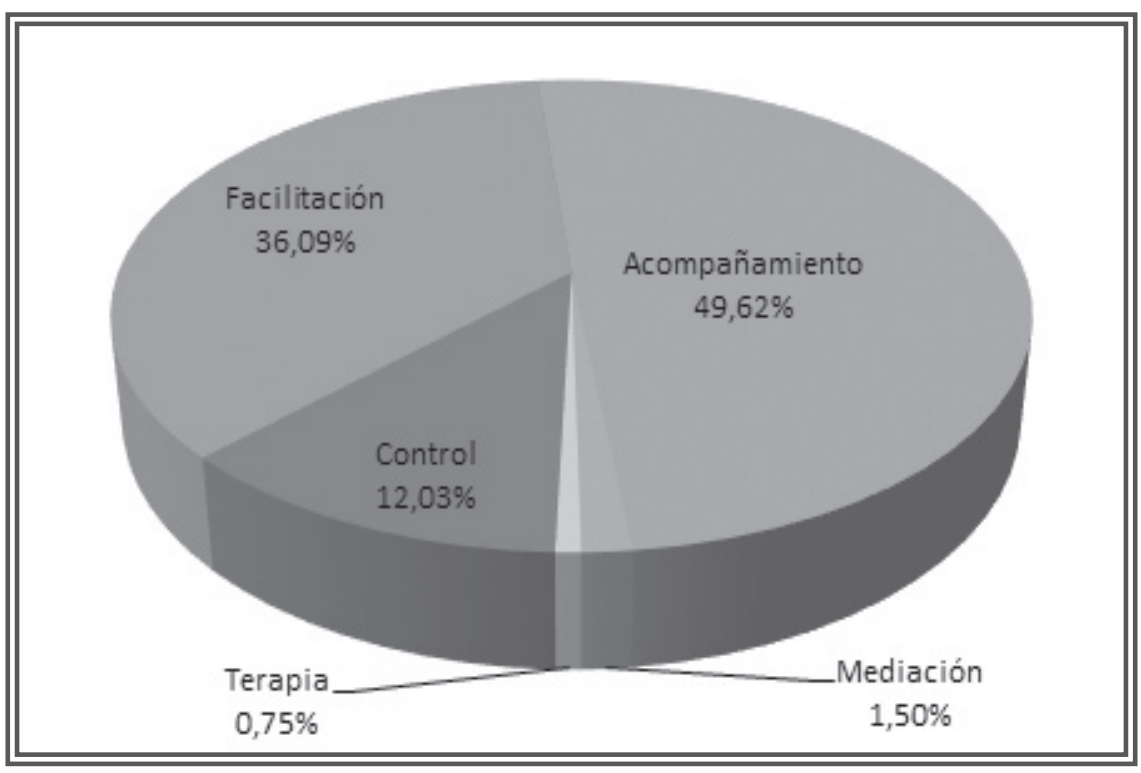

Figura 8. Distribución según el nivel de intervención Fuente: Elaboración de las investigadoras enfoca en las relaciones.

En la gráfica se evidencia que en la mayoría de los casos se realiza acompañamiento o sostén, seguido de facilitación y control, en este último, se activan las rutas de control definidas en la ciudad para los casos que lo ameritan. Además, en proporciones menores se da la mediación y la terapia, y aunque las familias pudieran beneficiarse de estas intervenciones, algunas veces no continúan cuando el paciente es dado de alta.

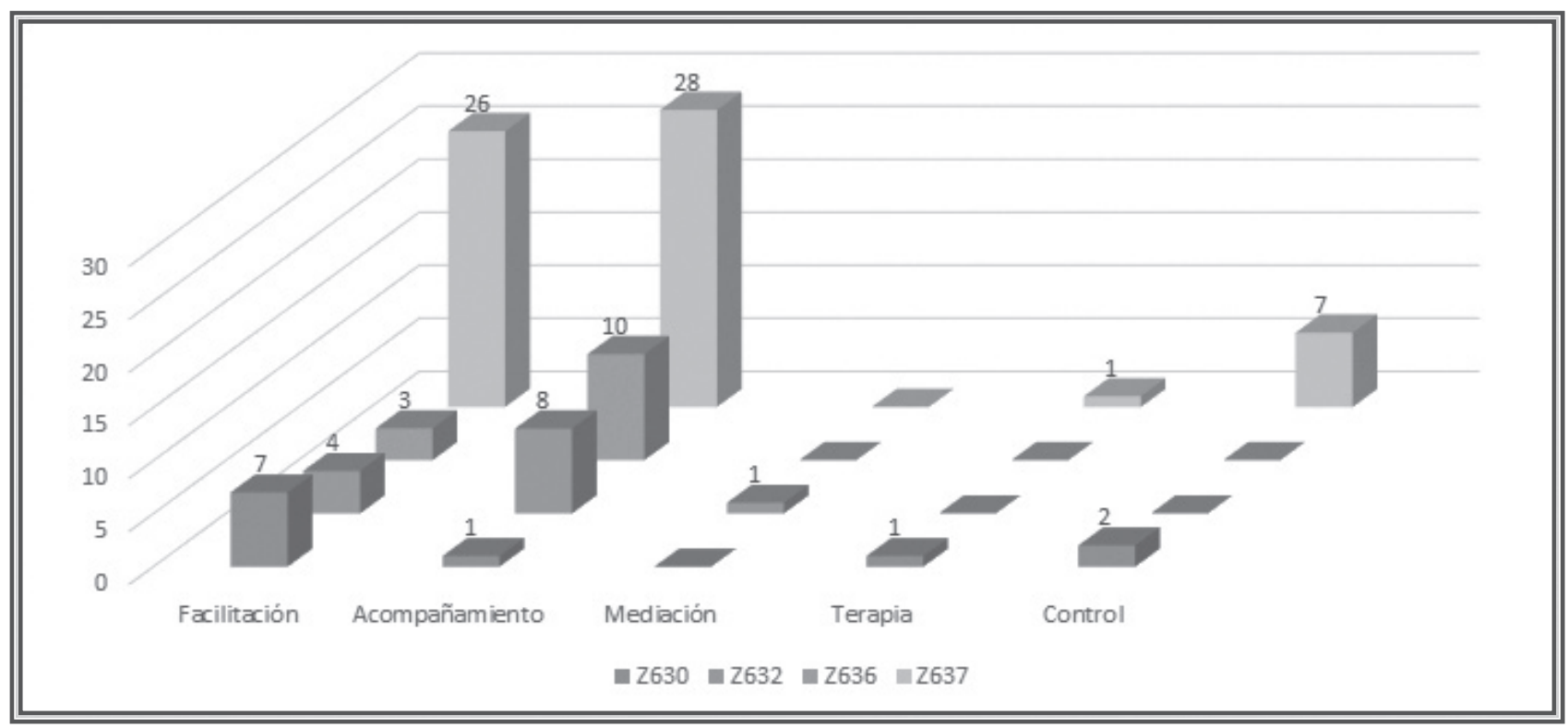

Figura 9. Distribución según diagnóstico y nivel de intervención Fuente: Elaboración de las investigadoras 


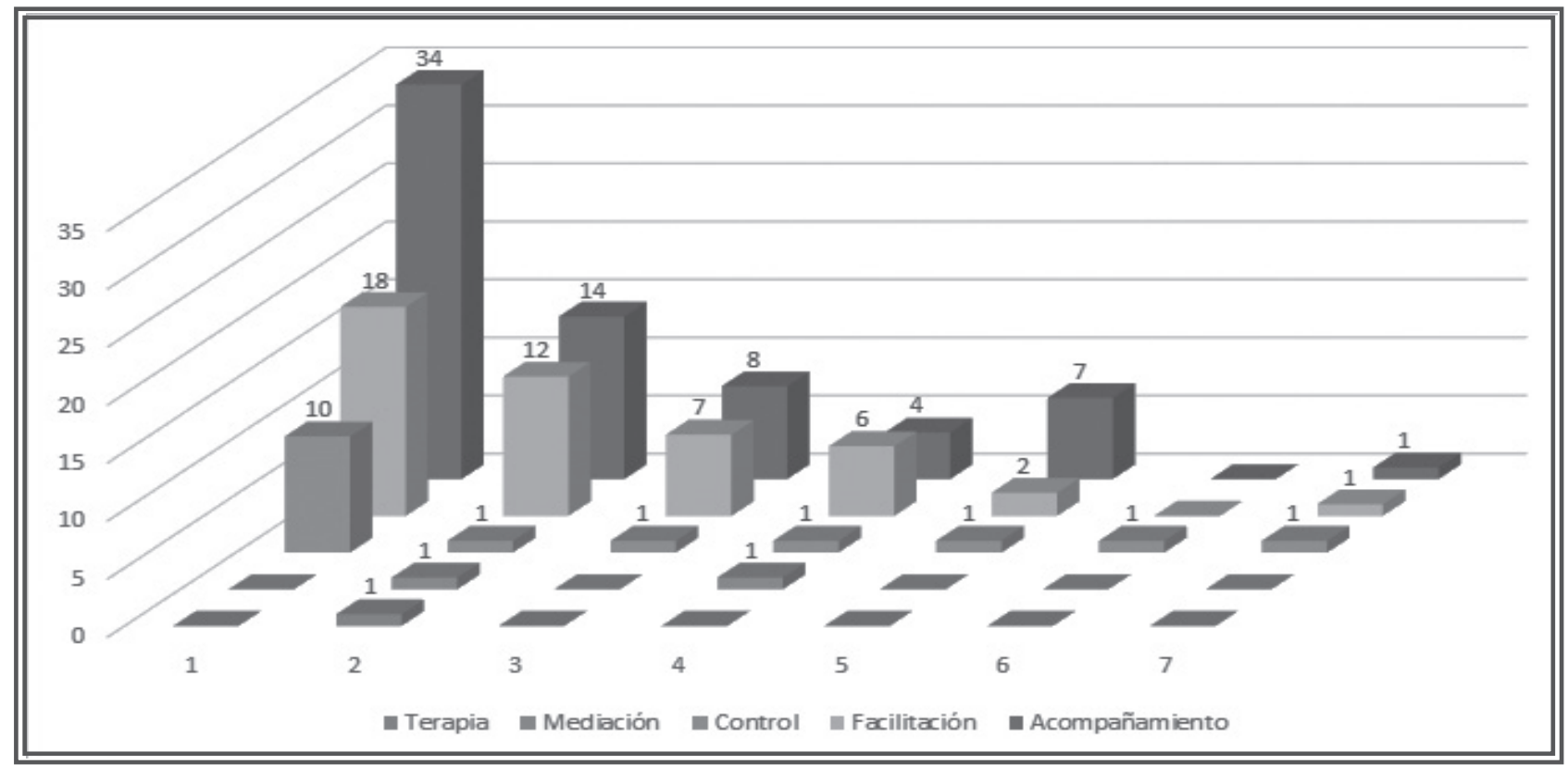

Figura 10. Relación entre el número de sesiones y el nivel de intervención Fuente: Elaboración de las investigadoras

La gráfica muestra que en los niveles de acompañamiento o sostén y facilitación se presentan los porcentajes más altos tanto para el diagnóstico de "problemas relacionados con hechos estresantes que afectan la familia (Z637) y para familiares dependientes de cuidado (Z636). En frecuencias menores, se encontró la mediación para abordar problemáticas de pareja (Z632) y el control y terapia en situaciones de apoyo inadecuado.

Esta gráfica permite reiterar que los tipos de intervención que realiza con mayor frecuencia Trabajo Social son el acompañamiento o sostén y la facilitación, predominando la atención en una sesión, aunque se registran casos entre 2 y 7 consultas.

\section{Soporte social y familiar}

on el objetivo de describir cómo se organizan las familias en sus
relaciones para responder a las necesidades de cuidado del paciente
durante su hospitalización, se revisaron las narraciones plasmadas por
las trabajadoras sociales en las historias clínicas sobre la atención realizada y
a partir de esto se formularon las siguientes subcategorías relacionadas con
condiciones y reacciones de las familias ante la situación de enfermedad, unas
que favorecen la adaptación a la misma y otras que la dificultan: Soporte
familiar y social; distribución de los roles; cohesión familiar; información y
comprensión de la enfermedad y del pronóstico; comunicación; espiritualidad 
y religiosidad; alteraciones emocionales; duelo anticipado; relación de pareja; condición económica; aislamiento social. Para efectos de este artículo haremos énfasis en el soporte familiar y social por ser la situación que más frecuentemente fue abordada por las trabajadoras sociales, y el motivo de consulta más solicitado por los médicos. Es importante aclarar que el soporte familiar se refiere al apoyo ofrecido por sus miembros y el social al dado por vecinos, cuidadores formales y el realizado por instituciones que por su misión o razón de ser les compete dicho acompañamiento.

\section{Cómo se organizan las familias para dar soporte al paciente}

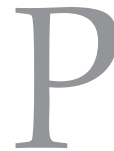

ara la mayoría de los familiares, el soporte no solo se considera como el apoyo emocional y físico ofrecido por ellos, sino que también incluye el aporte económico e instrumental (suministro de pañales, medicamentos, entre otros). Acompañar, visitar, turnarse o cumplir horarios mientras el paciente se encontraba en la clínica, son manifestaciones connotadas por las familias como apoyo moral.

"Entre varios hermanos colaboran ya sea presencialmente y otros hacen aportes económicos para la compra de pañales y demás insumos para el aseo y cuidado de él" (Código 60-7).

"Nosotros le hemos propuesto dos ideas para el cuidado: la primera es que mi hermana mayor se vaya a vivir con él para que esté pendiente y lo acompañe a todas partes; le dijimos que él se hiciera cargo del arrendamiento y que los hijos pagábamos los servicios, la alimentación y otros gastos" (Código 60-42).

Un caso en particular con respecto al soporte familiar, también permitió identificar cómo el cuidado del enfermo está relacionado con el tono afectivo que ha caracterizado las relaciones familiares que se han construido.

"Mi abuelo es quien siempre me ha cuidado, me brinda amor y está pendiente de mí y mis hermanos, él siempre quiere lo mejor para todos", "él, en este momento es mi prioridad más que mi hijo, yo al lado de mi abuelo viví muchas experiencias bonitas, esto ha sido lo más difícil para nosotros" (Código 60-4).

"Para mí está primero mi familia, en mi experiencia personal mi padre nos dejó porque mi mamá estaba muy enferma mentalmente entonces se 
aburrió y se le olvidó que también tenía un hijo, ahora yo no quiero repetir la historia (Código 60-49).

En lo que respecta al soporte social, se pudo observar que los familiares, para contribuir a la dignidad y estabilidad del enfermo, buscan por fuera del sistema familiar, diversos medios para brindarle un mejor apoyo. Inclusive de acuerdo a la situación económica por la que atraviesa la familia, se evidenció que en algunos casos se acude a la contratación de cuidadores formales, es decir, personas especializadas para cuidar del enfermo; en otros, se acude al vecino o a las amistades que colaboran sin recibir a cambio una remuneración (cuidador informal).

"El patrón me va a cuidar hasta que pueda trabajar, él se va a encargar de mí, va a venir por mí para irnos a la finca" (Código 34-7).

"Cuando tengo que dejarla sola, le pago a una señora para que la cuide todo el tiempo, o si no, le digo a las vecinas que me colaboren, intento nunca dejarla sola" (Código 34-23).

"Me visita una amiga cada tres días para traerme ropa y acompañarme" (Código 34-3).

Por otro lado, en los casos que requirieron algún tipo de intervención para reestablecer los derechos del paciente, se encontró que tanto la institución de salud como las familias implicadas, buscaron hacer efectiva esta protección, iniciando un proceso continuo de atención a la problemática referida. Dentro de esta, se halló que la clínica brinda orientación y asesoría a los familiares, facilitando así la participación en los programas establecidos dentro de la misma. Además, en algunos casos, las familias ya tenían avances en este proceso al momento de la hospitalización.

"Ya el caso está siendo investigado en la Fiscalía y hemos recibido ayuda de psicólogo, trabajador social del Hospital General y de la Policía, nos han ayudado mucho" (Código 34-14).

\section{Cómo se organizan cuando no hay un soporte efectivo}

\footnotetext{
n los pacientes que no cuentan con un soporte familiar, el hecho de tener amigos y entablar nuevas relaciones durante su estadía en la clínica, Jujuega un papel importante, puesto que se construye una relación de cuidado mutuo.
} 
"Yo veo que ella no se está alimentando adecuadamente, le digo que tiene que alimentarse para recuperarse" (Código 34-34).

La falta del soporte familiar emocional, físico, económico y social por parte de la familia, es motivo de interconsulta por parte de los médicos a Trabajo Social. La falta de acompañamiento al paciente durante su hospitalización por parte de sus familiares, fue explicada por la mayoría de estos refiriendo una diversidad de obligaciones, que les impide estar pendientes del enfermo, tales como velar por su familia nuclear, contar con un trabajo que por sus horarios o ubicación geográfica no les permite compartir con el paciente, y asumir los gastos económicos que genera visitarlo.

"Mi trabajo no es muy estable y me queda muy difícil dar dinero en la casa de mi mamá, yo tengo que velar por mi hija y mi esposa, y si vengo una horas a la semana me sale muy costoso, tengo que utilizar doble bus tanto de ida como de vuelta" (Código 26-13).

Adicionalmente, la ausencia de familiares llevó a que en el momento del alta, el paciente no contara con la ayuda efectiva para continuar con su proceso de cuidado en casa. Esto se presenta ya sea porque los pacientes tienen familiares lejos de su residencia o porque las relaciones familiares son distantes, con poca comunicación o con conflictos marcados y pobre cohesión.

"En mi familia no encuentro ayuda, mi mamá siempre ha sido muy distante y con mis tres hermanos no tenemos ningún contacto" (Código 26-4).

"Yo no tengo hijos, ninguno se preocupa por mí, no ve que un día mi hija menor se fue de la casa y volvió porque no tenía dinero para pagar el arriendo" (Código 26-11).

Otras situaciones que hablan de la falta del soporte familiar, en cuanto a los aspectos instrumentales y físicos, son las dificultades para ser efectivos en diversas acciones que se requieren para el tratamiento del paciente, como por ejemplo solicitar citas médicas, hacer un acompañamiento emocional para ayudar a la persona a enfrentar la situación, velar por el cuidado e higiene, brindar la alimentación apropiada y suministrar la medicación que el enfermo necesita. Se puede señalar, que en algunos casos, esta situación recae en la responsabilidad del cuidador principal o del mismo paciente. 
"Antes del accidente no nos dejaba entrar a la casa ni verla, el día que se accidentó que nos llamó cuando fuimos, vimos que estaba en condiciones deplorables, las uñas eran largas, mugre en el cabello y entre las uñas, escamas en el cuerpo, usted sabe eso es puro descuido "(llanto)", (Código 2-3).

Algunos pacientes manifestaron querer contar con un soporte familiar, que les ayude con el cuidado de su hogar y sus hijos, pues el no tener quien cuide de ellos les ocasiona mayor preocupación, mostrándose tristes, intranquilos o molestos y con la sensación de soledad, pues se sienten sin amor y sin afecto.

"Desde pequeña siempre he sentido el rechazo de mi madre", "ella me pide dinero, pero como yo no le puedo dar, se enoja y me sigue rechazando", "le pedí que se quedara con los niños mientras que yo estaba aquí y prefirió irse para la casa de otra hija" (llora), (Código 26-20).

Solo en una historia se narró una dificultad específica de la falta de soporte social, por los obstáculos presentados con la aseguradora en la realización del tratamiento necesario para el paciente, por lo que los familiares deciden descartar dicha opción y hacerlo de manera particular.

"La madre refiere que hace seis años la paciente no recibe tratamiento debido a dificultades con la EPS "allá son muy demorados y nunca asignan una cita además yo llevo a la niña al médico y lo máximo que le mandan es un examen de orina" (Código 2-1).

Otra forma de expresar dificultades en este ámbito, se refiere al distanciamiento que el paciente asume respecto a los recursos sociales, aislándose de su entorno. Se encontraron dos situaciones: En uno de ellos, la enfermedad lo llevó a que se aislara de su entorno social, pues al no poder realizar actividades recreativas y sociales le impedía compartir con sus grupos sociales.

"Yo me mantengo encerrada, salgo a ponerme la inyección y ya, me da pena que mis amigos me vean hacerlo y no siempre estoy bien para salir" (Código 26-2).

Otra situación describe la impotencia de la familia para encontrar estrategias relacionales con su ser querido.

"Todos hemos venido a visitarlo, pero él se mantiene dormido, entonces para que venimos si no nos habla, ni nos ve" (Código 26-7). 


\section{Conclusiones}

e revisaron 133 historias de pacientes hospitalizados en la CUB remitidos por alguno de los profesionales del equipo de salud a trabajo social en el periodo comprendido entre febrero y julio del 2013. El principal diagnóstico de las familias corresponde a "hechos estresantes que afectan la familia", lo cual evidencia una serie de situaciones que generan tensiones, angustia, sufrimiento y deseos de recibir una atención profesional que les permita enfrentar la enfermedad y las situaciones conexas que afectan el bienestar y la armonía familiar.

La intervención familiar que se ofrece desde el servicio de Trabajo Social, tiene como propósito atender a las familias mediante el acompañamiento y facilitación para que cumplan la función de soporte del paciente en su proceso de enfermedad, hospitalización y recuperación o, en algunos casos, en el período final de su vida. En la mayoría de los casos, las familias asisten a una consulta con Trabajo Social lo cual, constituye una oportunidad de ser escuchadas en sus sentimientos e inquietudes, orientadas en cuanto a los requerimientos del paciente y a su tratamiento.

Se pudo establecer mediante los testimonios consignados por las profesionales de trabajo social en la historia clínica, que las familias reaccionan de diversas formas en cuanto al soporte y apoyo que requieren los pacientes. Si bien, lo más frecuente es encontrar familias que se interesan en el tratamiento y participan con su afecto y su apoyo material en las diferentes fases, hay algunas que se muestran distantes o reacias por razones objetivas (trabajo, dificultades económicas) o por razones subjetivas (sentimientos negativos hacia el paciente y al tratamiento casi siempre por historias no gratas que han vivido a su lado).

\section{BIBLIOGRAFÍA}

Estrada Arango, Piedad. (2006). La familia de hoy: sus tipologías y conflictos. En: Observatorio para la Equidad y la Integración Social en Medellín y Antioquia. Medellín. (15), 8-11.

Flórez, Carmen Elisa y Sánchez, Lina María. (2013). Fecundidad y familia en Colombia: ¿Hacia una segunda transición demográfica? Encuesta Nacional de Salud. 1990 - 2010. Profamilia. http:// wWw.profamilia.org.co/encuestas/Profamilia/Profamilia/images/stories/ENDS\%201990\%20 -2010/Estudio\%20a\%20profundidad\%20-\%20Fecundidad\%20y\%20familia\%20-\%20Final.pdf Fecha de consulta 10 de nov de 2015.

Fruggeri, Laura. (2002). "Una propuesta de clasificación de las intervenciones en favor de la familia: de los contenidos a los procesos". En: Sistemas familiares. Argentina. Asociación Sistémica de Buenos Aires. (1-2), 5-13.

Galeano Marín, María Eumelia. (2004). Diseño de proyectos de investigación cualitativa. Medellín Colombia: Fondo Editorial Universidad Eafit. 82 Págs. 